\title{
Valor do Diâmetro do Átrio Esquerdo com Escore CHA2DS2-Vasc na Predição da Trombose Atrial Esquerda/Trombose de Apêndice Atrial Esquerdo na Fibrilação Atrial Não Valvar
}

\author{
Value of Left Atrial Diameter with CHA2DS2-VASc Score in Predicting Left Atrial/Left Atrial Appendage \\ Thrombosis in Non-valvular Atrial Fibrillation
}

Yu Zhang ${ }^{1}$ e Yi-Qiang Yuan ${ }^{10}$

Cardiovascular Hospital of Zhengzhou, ${ }^{1}$ Zhengzhou - China

\section{Resumo}

Fundamentos: A fibrilação atrial é a arritmia persistente mais comum e é o principal fator que leva ao tromboembolismo.

Objetivo: Investigar o valor do diâmetro do átrio esquerdo combinado com o escore CHA2DS2-VASc na predição da trombose atrial esquerda/trombose de apêndice atrial esquerdo na fibrilação atrial não valvar.

Métodos: Trata-se de estudo retrospectivo. 238 pacientes com fibrilação atrial não valvar foram selecionados e divididos em dois grupos: trombose e não trombose. Determinou-se o escore CHA2DS2-VASc. Valores de $\mathrm{p}<0,05$ foram considerados estatisticamente significativos.

Resultados: A análise de regressão logística multivariada revelou que histórico de acidente vascular cerebral/ataque isquêmico transitório, doença vascular, escore CHA2DS2-VASc, DAE, DDFVE e FEVE foram fatores de risco independentes para trombose atrial esquerda/trombose de apêndice atrial esquerdo $(\mathrm{p}<0,05)$. A análise da curva ROC (Receiver Operating Characteristic) revelou que a área sob a curva para o escore CHA2DS2-VASc na predição de trombose atrial esquerda/trombose de apêndice atrial esquerdo foi de 0,593 quando o escore CHA2DS2-VASc foi $\geq 3$ pontos, e a sensibilidade e especificidade foram $86,5 \%$ e $32,6 \%$, respectivamente, enquanto a área sob a curva para o DAE na predição de trombose atrial esquerda/trombose de apêndice atrial esquerdo foi 0,786 quando o DAE foi $\geq 44,17 \mathrm{~mm}$, e a sensibilidade e especificidade foram $89,6 \%$ e $60,9 \%$, respectivamente. Entre os diferentes grupos CHA2DS2-VASc, a taxa de incidência de trombose atrial esquerda/trombose de apêndice atrial esquerdo em pacientes com DAE $\geq 44,17 \mathrm{~mm}$ foi maior do que em pacientes com DAE $<44,17 \mathrm{~mm}(p<0,05)$.

Conclusão: O escore CHA2DS2-VASc e o DAE estão correlacionados com a trombose atrial esquerda/trombose de apêndice atrial esquerdo na fibrilação atrial não valvar. Para pacientes com escore CHA2DS2-VASc de 0 ou 1, quando o DAE é $\geq 44,17$ $\mathrm{mm}$, o risco de trombose atrial esquerda/trombose de apêndice atrial esquerdo permaneceu alto. (Arq Bras Cardiol. 2021; 116(2):325-331)

Palavras-chave: Fibrilação Atrial Não Valvar; Acidente Vascular Cerebral; Avaliação de Risco; Escore de Propensão; Átrio Esquerdo; Apêndice Atrial; Trombose.

\begin{abstract}
Background: Atrial fibrillation is the most common persistent arrhythmia, and is the main factor that leads to thromboembolism.

Objective: To investigate the value of left atrial diameter combined with CHA2DS2-VASC score in predicting left atrial/left atrial appendage thrombosis in non-valvular atrial fibrillation.

Methods: This is a retrospective study. 238 patients with non-valvular atrial fibrillation were selected and divided into two groups: thrombosis and non-thrombosis. CHA2DS2-VASC score was determined. $P<0.05$ was considered statistically significant.

Results: Multivariate logistic regression analysis revealed that the history of stroke/transient ischemic attack, vascular disease, CHA2DS2-VASC score, left atrial diameter (LAD), left ventricular end-diastolic dimension (LVEDD) and left ventricular ejection fraction (LVEF) were independent risk factors for left atrial/ left atrial appendage thrombosis $(p<0.05)$. Receiver operating characteristic curve analysis revealed that the area under the curve for the CHA2DS2-VASC score in predicting left atrial/left atrial appendage thrombosis was 0.593 when the CHA2DS2-VASC score was $\geq 3$ points, and sensitivity and specificity were $86.5 \%$ and $32.6 \%$, respectively, while the area under the curve for LAD in predicting left atrial/left atrial appendage thrombosis was 0.786 when LAD was $\geq 44.17 \mathrm{~mm}$, and sensitivity and specificity were $89.6 \%$ and $60.9 \%$, respectively. Among the different CHA2DS2-VASc groups, the incidence rate of left atrial/left atrial appendage thrombosis in patients with $L A D \geq 44.17 \mathrm{~mm}$ was higher than patients with $L A D<44.17 \mathrm{~mm}(p<0.05)$.
\end{abstract}

Correspondência: Yi-Qiang Yuan •

Cardiovascular Hospital of Zhengzhou - Cardiolgy - No.17 Jingnan 5 Street, Economic \& Technological District Zhangzhou 450000 China Zhangzhou 450000 - China E-mail: yuanyq639841@163.com

Artigo recebido em 26/07/2019, revisado em 15/11/2019, aceito em 27/12/2019

DOI: https://doi.org/10.36660/abc.20190492 
Conclusion: CHA2DS2-VASC sCore and LAD are correlated with left atrial/left atrial appendage thrombosis in non-valvular atrial fibrillation. For patients with a CHA2DS2-VASC score of 0 or 1, when LAD is $\geq 44.17 \mathrm{~mm}$, the risk for left atrial/left atrial appendage thrombosis remained high. (Arq Bras Cardiol. 2021; 116(2):325-331)

Keywords: Atrial Fibrillation Non Valvar; Stroke; Risk Assessment; Propensity Score; Heart Atria; Atrial Appendage.

Full texts in English - http://www.arquivosonline.com.br

\section{Introdução}

A fibrilação atrial (FA) é a arritmia persistente mais comum e é o principal fator que leva ao tromboembolismo. ${ }^{1}$ Nos últimos anos, com o envelhecimento da população na China, a incidência dessa doença aumentou. ${ }^{2}$ Portanto, essa doença representa uma séria ameaça à vida e à saúde das pessoas. Quando a FA ocorre, o átrio cardíaco não pode se contrair regularmente e efetivamente e o fluxo sanguíneo diminui, o que aumenta muito o risco de trombose atrial esquerda/ trombose de apêndice atrial esquerdo, ${ }^{3}$ e a trombose atrial esquerda/trombose de apêndice atrial esquerdo aumenta ainda mais o risco de episódios de tromboembolismo. ${ }^{4}$ Portanto, a avaliação científica da trombose atrial esquerda/ trombose de apêndice atrial esquerdo é de grande importância para orientar o tratamento e melhorar o prognóstico dos pacientes. O CHA2DS2-VASc é um escore atualmente e amplamente usado para avaliar o risco de acidente vascular cerebral em pacientes com FA não valvar, e desempenha um papel importante na determinação de fatores de alto risco e orientação do tratamento. ${ }^{5}$ No entanto, o escore depende principalmente do registro do histórico do paciente. Um estudo revelou que ${ }^{6}$ o tamanho do átrio esquerdo estava intimamente relacionado à trombose atrial esquerda/trombose de apêndice atrial esquerdo. No entanto, não está claro se o diâmetro do átrio esquerdo combinado com o escore CHA2DS2-VASc pode melhorar os resultados preditivos de trombose atrial esquerda/trombose de apêndice atrial esquerdo. O objetivo deste estudo foi analisar os fatores relacionados à trombose atrial esquerda/trombose de apêndice atrial esquerdo em pacientes com FA não valvar e explorar o valor do diâmetro do átrio esquerdo combinado com o escore CHA2DS2-VASc na predição de trombose atrial esquerda/trombose de apêndice atrial esquerdo, a fim de fornecer referências para a prática clínica.

\section{Dados e métodos}

\section{Dados gerais}

Trata-se de um estudo retrospectivo. Os dados foram coletados em prontuários médicos. No total, 238 pacientes com FA não valvar, hospitalizados no Zhengzhou Cardiovascular Hospital de fevereiro de 2012 a março de 2017, foram incluídos no estudo. Critérios de inclusão: (1) pacientes diagnosticados por eletrocardiograma (ECG) ou ECG dinâmico; (2) pacientes submetidos à ecocardiografia transesofágica. Critérios de exclusão: (1) pacientes com cardiopatia reumática, FA valvar e FA paroxística; (2) pacientes com infarto agudo do miocárdio e insuficiência cardíaca aguda descompensada em 90 dias e pacientes com histórico de cirurgia cardíaca; (3) pacientes com embolia pulmonar, trombose venosa profunda, histórico de administração de anticoagulantes, como varfarina e rivaroxabana, ou hipolipemiantes, como estatinas; (4) pacientes com tumores malignos, hipertireoidismo e disfunção hepática e renal grave. Esse estudo foi aprovado pelo Comitê de Ética do nosso hospital. Todos os pacientes forneceram consentimento informado assinado.

\section{Método}

\section{Aquisição de dados clínicos}

Foram coletadas as seguintes informações de todos os pacientes: sexo, idade, curso da FA, tabagismo e alcoolismo, histórico de doença crônica, altura e peso. Calculou-se o índice de massa corporal (IMC). Além disso, glicemia em jejum (GJ), colesterol total (CT), triglicerídeos (TG), colesterol de lipoproteína de baixa densidade (LDL-c), colesterol de lipoproteína de alta densidade (HDL-c), contagem de plaquetas $(\mathrm{Plt})$, ácido úrico sérico $(\mathrm{AU})$ e outros indicadores bioquímicos foram coletados.

\section{Ecocardiografia transtorácica e ecocardiografia transesofágica}

Todos os exames foram realizados por um ultrassonografista experiente com o título de Médico Chefe em nosso hospital. Todos os pacientes assinaram o termo de consentimento informado antes do exame. Para realizar o exame, utilizou-se um aparelho diagnóstico de ultrassom Doppler colorido Philips iE33. Realizou-se ecocardiografia transtorácica de rotina, e a frequência da sonda foi de $2,5 \mathrm{MHz}$. Foram medidos o diâmetro atrial esquerdo (DAE), a dimensão diastólica final do ventrículo esquerdo (DDFVE) e a fração de ejeção do ventrículo esquerdo (FEVE). Posteriormente, administrouse anestesia faríngea local com lidocaína. A seguir, a sonda foi colocada no esôfago até a localização do coração, e a frequência da sonda foi de 5,0 MHz. As seções do átrio esquerdo e do apêndice atrial esquerdo foram continuamente observadas para determinar se o trombo estava presente no átrio esquerdo/apêndice atrial esquerdo. Esses pacientes foram divididos em dois grupos: grupo trombose e grupo não trombose.

\section{Escore CHA2DS2-VASc}

O escore CHA2DS2-VASc foi calculado de acordo com os dados clínicos básicos dos pacientes: ${ }^{7}$ (1) principais fatores de risco (2 pontos por item): idade $\geq 75$ anos, AVC isquêmico e ataque isquêmico transitório; (2) fatores secundários (1 ponto por item): mulheres, com idades entre 65 e 74 anos, hipertensão, diabetes, doença vascular e insuficiência cardíaca crônica; (3) escore mais baixo de 0 e escore mais alto de 9. Quanto maior o escore, maior a possibilidade de trombose. 


\section{Análise estatística}

Para o agrupamento de dados e análises estatísticas, utilizou-se o programa estatístico SPSS versão 21.0. Os dados contínuos foram expressos como média \pm desvio padrão $(\mathrm{x} \pm \mathrm{DP})$ e comparados entre dois grupos usando o teste $t$ de Student não pareado de distribuição normal, e o teste de Kolmogorov-Smirnov (K-S) foi usado para distribuição normal. Os dados categóricos foram expressos em taxas (\%) e comparados entre dois grupos usando o teste X2. Realizou-se a análise de regressão logística multivariada para analisar os fatores relacionados que afetaram a trombose atrial esquerda/ trombose de apêndice atrial esquerdo. Utilizou-se a curva ROC para analisar os resultados preditivos do diâmetro atrial esquerdo e do escore CHA2DS2-VASc para trombose atrial esquerda/trombose de apêndice atrial esquerdo. Valores de $p<0,05$ foram considerados estatisticamente significativos.

\section{Resultados}

\section{Trombose atrial esquerda/trombose de apêndice atrial esquerdo}

No total, 238 pacientes com FA não valvar foram incluídos neste estudo. Entre esses pacientes, 151 pacientes eram do sexo masculino e 87 do sexo feminino, e a idade desses

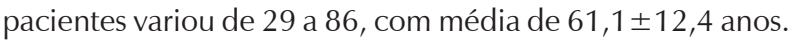
Nesses 238 pacientes, ocorreu trombose atrial esquerda/ trombose de apêndice atrial esquerdo em 46 pacientes, e a incidência foi de 19,3\%.

\section{Comparação de dados clínicos entre o grupo trombose e o grupo não trombose}

Diferenças de sexo, IMC, curso de FA, proporções de pacientes com histórico de tabagismo e consumo de álcool, proporções de pacientes com diabetes e doença cardíaca coronariana, escore CHA2DS2-VASc, GJ, CT, TG, LDL-c, Plt, $\mathrm{AU}$ e terapia medicamentosa entre o grupo trombose e o grupo não trombose não foram estatisticamente significativas $(p>0,05)$. No grupo trombose, a proporção de pacientes com idade $\geq 75$, a proporção de pacientes com hipertensão, a proporção de pacientes com insuficiência cardíaca, a proporção de pacientes com histórico de acidente vascular cerebral/ataque isquêmico transitório, a proporção de pacientes com histórico de doença vascular e o escore CHA2DS2-VASc, DAE e DDFVE foram maiores do que aqueles no grupo não trombose, enquanto o HDL-c e FEVE foram menores do que aqueles no grupo não trombose, e todas as diferenças foram estatisticamente significativas $(\mathrm{p}<0,05$, Tabela 1).

\section{Fatores relacionados que afetam a trombose atrial esquerda/trombose de apêndice atrial esquerdo}

Com a determinação de se a trombose atrial esquerda/ trombose de apêndice atrial esquerdo existia como uma variável dependente, e as variáveis com um valor de $p<0,10$ como variáveis independentes, realizou-se análise de regressão logística multivariada. Os resultados revelaram que o histórico de acidente vascular cerebral/ataque isquêmico transitório, doença vascular, escore CHA2DS2-VASc, DAE, DDFVE e FEVE foram fatores de risco independentes para trombose atrial esquerda/trombose de apêndice atrial esquerdo ( $p<0,05$, Tabela 2$)$.

\section{0 valor do DAE e do escore CHA2DS2-VASc na predição de trombose atrial esquerda/trombose de apêndice atrial esquerdo}

A análise da área sob a curva ROC revelou que quando o escore CHA2DS2-VASc foi usado para predizer a trombose atrial esquerda/trombose de apêndice atrial esquerdo, a área sob a curva foi de 0,593 (IC 95\%: 0,495-0.690). Quando o escore CHA2DS2-VASc foi $\geq 3$, a sensibilidade e a especificidade foram de $86,5 \%$ e $32,6 \%$, respectivamente. Quando o DAE foi usado para prever trombose atrial esquerda/trombose de apêndice atrial esquerdo, a área sob a curva foi de 0,786 (IC de 95\%: 0,704-0,868). Quando o DAE foi $\geq 44,17 \mathrm{~mm}$, a sensibilidade e a especificidade foram $89,6 \%$ e $60,9 \%$, respectivamente (Figura 1 ).

\section{Efeito do DAE no risco de trombose atrial esquerda/ trombose de apêndice atrial esquerdo em pacientes nos diferentes grupos CHA2DS2-VASc}

Nos diferentes grupos CHA2DS2-VASc, a incidência de trombose atrial esquerda/trombose de apêndice atrial esquerdo em pacientes com DAE $\geq 44,17 \mathrm{~mm}$ foi maior do que em pacientes com DAE $<44,17 \mathrm{~mm}$, e a diferença foi estatisticamente significativa $(p<0,05$, Tabela 3$)$.

\section{Discussão}

Como o tipo de arritmia mais comum em Departamentos de Clínica Médica Cardiovascular, a FA é um fator de risco que leva ao tromboembolismo ${ }^{8}$. Em comparação com a população não FA, o risco de AVC em pacientes com FA aumenta cinco vezes. ${ }^{9}$ Além disso, um estudo revelou que ${ }^{10}$ o trombo que causou AVC em pacientes com FA veio principalmente do átrio esquerdo/apêndice atrial esquerdo. A trombose atrial esquerda/trombose de apêndice atrial esquerdo é um fator de risco independente para acidente vascular cerebral em pacientes com FA não valvar. ${ }^{11}$ Isso pode aumentar significativamente o risco de eventos tromboembólicos e é um indicador direto de terapia anticoagulante em pacientes com FA. ${ }^{12}$ Portanto, a detecção precoce da trombose atrial esquerda/trombose de apêndice atrial esquerdo ou fatores de alto risco para trombose atrial esquerda/trombose de apêndice atrial esquerdo é de grande importância para orientar o tratamento e melhorar o prognóstico de pacientes com FA. Neste estudo, 238 pacientes com FA, que não receberam anticoagulação e terapia hipolipemiante, foram incluídos. Os resultados da ecocardiografia transesofágica revelaram que a incidência de trombose atrial esquerda/trombose de apêndice atrial esquerdo foi de 19,3\%. Essa porcentagem se assemelha aos $18,6 \%$ relatados por Shuanglun Xie et al., ${ }^{13}$ e aos 20,7\% relatados por Weiwei $\mathrm{Fu}$ et al. ${ }^{14}$ Esses resultados revelam que a incidência de trombose atrial esquerda/trombose de apêndice atrial esquerdo é relativamente alta em pacientes com FA sem anticoagulação e terapia hipolipemiante. 


\begin{tabular}{|c|c|c|c|c|}
\hline Índice & $\begin{array}{l}\text { Grupo trombose } \\
\qquad(n=46)\end{array}$ & $\begin{array}{c}\text { Grupo não } \\
\text { trombose }(n=192)\end{array}$ & $t / X^{2}$ & $\mathrm{p}$ \\
\hline \multicolumn{5}{|l|}{ Idade (n, \%) } \\
\hline$<65$ anos & $18(39,1)$ & $112(58,3)$ & & \\
\hline $65-74$ anos & $15(32,6)$ & $62(32,3)$ & & \\
\hline$\geq 75$ anos & $13(28,3)$ & $18(9,4)$ & 12,668 & 0,002 \\
\hline Sexo (M/F) & $32(69,6)$ & $119(62,0)$ & 0,921 & 0,337 \\
\hline IMC $\left(\mathrm{kg} / \mathrm{m}^{2}\right)$ & $26.82 \pm 3,70$ & $25.94 \pm 3,01$ & 1,696 & 0,091 \\
\hline Anos de $A F(A)$ & $4.69 \pm 1,69$ & $5.10 \pm 1,38$ & 1,718 & 0,087 \\
\hline Tabagismo (n, \%) & $18(39,1)$ & $71(37,0)$ & 0,921 & 0,337 \\
\hline Alcoolismo (n, \%) & $11(23,9)$ & $36(18,8)$ & 0,624 & 0,429 \\
\hline Hipertensão arterial $(n, \%)$ & $32(69,6)$ & $91(47,4)$ & 7,304 & 0,007 \\
\hline Diabetes mellitus $(\mathrm{n}, \%)$ & $8(17,4)$ & $44(22,9)$ & 0,664 & 0,415 \\
\hline Doença coronariana $(n, \%)$ & $4(8,7)$ & $8(4,2)$ & 1,590 & 0,207 \\
\hline Insuficiência cardíaca, (n, \%) & $7(15,2)$ & $6(3,1)$ & 10,508 & 0,001 \\
\hline Acidente vascular cerebral/Ataque isquêmico transitório (n, \%) & $17(37,0)$ & $11(5,7)$ & 101,138 & 0,000 \\
\hline Doença vascular (n, \%) & $22(47,8)$ & $51(26,6)$ & 7,890 & 0,005 \\
\hline $\mathrm{GJ}(\mathrm{mmol} / \mathrm{L})$ & $5,72 \pm 0,86$ & $6,13 \pm 1,43$ & 1,832 & 0,068 \\
\hline $\mathrm{CT}(\mathrm{mmol} / \mathrm{L})$ & $4,82 \pm 0,96$ & $4,66 \pm 0,98$ & 1,036 & 0,301 \\
\hline TG $(\mathrm{mmol} / \mathrm{L})$ & $1,84 \pm 1,02$ & $1,68 \pm 0,92$ & 1,055 & 0,292 \\
\hline LDL-c (mmol/L) & $3,00 \pm 0,54$ & $2,96 \pm 0,86$ & 0,298 & 0,766 \\
\hline $\mathrm{HDL}-\mathrm{c}(\mathrm{mmol} / \mathrm{L})$ & $0,99 \pm 0,18$ & $1,16 \pm 0,31$ & 3,458 & 0,001 \\
\hline Plt $\left(\times 10^{9} / L\right)$ & $209,08 \pm 34,45$ & $214,43 \pm 41,26$ & 0,815 & 0,416 \\
\hline $\mathrm{AU}(\mu \mathrm{mol} / \mathrm{L})$ & $333,70 \pm 64,68$ & $342,74 \pm 70,08$ & 0,798 & 0,426 \\
\hline Escore CHA2DS2-VASc & $2,26 \pm 1,90$ & $1,64 \pm 1,48$ & 2,428 & 0,016 \\
\hline Grupo CHA2DS2-VASc (n, \%) & & & 2,635 & 0,268 \\
\hline Escore 0 & $8(17,4)$ & $55(28,6)$ & & \\
\hline Escore 1 & $14(30,4)$ & $56(29,2)$ & & \\
\hline Escores $\geq 2$ & $24(52,2)$ & $81(42,2)$ & & \\
\hline $\mathrm{DAE}(\mathrm{mm})$ & $45,81 \pm 6,16$ & $38,55 \pm 5,00$ & 6,118 & 0,000 \\
\hline DDFVE (mm) & $51,35 \pm 4,38$ & $48,53 \pm 4,11$ & 4,133 & 0,000 \\
\hline FEVE (\%) & $57,05 \pm 10,50$ & $61,84 \pm 9,17$ & 3,092 & 0,002 \\
\hline \multicolumn{5}{|l|}{ Tratamento medicamentoso $(\mathrm{n}, \%)$} \\
\hline Betabloqueadores & $15(32,6)$ & $54(28,1)$ & 0,362 & 0,547 \\
\hline IECA/BRA & $21(45,7)$ & $75(39,1)$ & 0,670 & 0,413 \\
\hline
\end{tabular}

MC: indice de massa corporal; GJ: glicemia em jejum; CT: colesterol total; TG: triglicerídeos; LDL-c: colesterol de lipoproteina de baixa densidade; HDL-C: colesterol de lipoproteína de alta densidade; Plt: contagem de plaquetas; AU: ácido úrico sérico; DAE: diâmetro atrial esquerdo; DDFVE: dimensão diastólica final do ventrículo esquerdo; FEVE: fração de ejeção do ventrículo esquerdo; IECA: inibidor da enzima de conversão da angiotensina; BRA: bloqueadores dos receptores de angiotensina.

O escore CHA2DS2-VASc foi estabelecido otimizando ainda mais o escore CHADS2, que é um método clínico comumente usado para avaliar o risco de acidente vascular cerebral em pacientes com FA atualmente, sendo também usado para orientar o tratamento clínico. ${ }^{15} \mathrm{Um}$ estudo revelou que ${ }^{16}$ um escore $\mathrm{CHA} 2 \mathrm{DS} 2-\mathrm{VASc} \geq 2$ é um fator de risco independente para trombose atrial esquerda/ trombose de apêndice atrial esquerdo em pacientes com FA. O presente estudo revelou que o escore CHA2DS2-
VASc foi maior no grupo trombose do que no grupo não trombose. No entanto, a diferença na distribuição dos escores CHA2DS2-VASc entre os dois grupos não foi estatisticamente significativa. A análise univariada e multivariada revelou que o escore CHA2DS2-VASc é um fator de risco independente para trombose atrial esquerda/ trombose de apêndice atrial esquerdo. Além disso, esses resultados revelam que o escore $\mathrm{CHA} 2 \mathrm{DS} 2-\mathrm{VASc}$ está correlacionado com a trombose atrial esquerda/trombose 
Artigo Original

\begin{tabular}{lccccc}
\hline \multicolumn{7}{l}{ Tabela 2 - Fatores relacionados a trombo em átrio esquerdo ou apêndice atrial esquerdo } & & \\
\hline Índice & $\mathbf{B}$ & EP & Wals $\mathbf{2}^{2}$ & $\mathbf{p}$ & OR (Cl: 95\%) \\
\hline AVC/Ataque isquêmico transitório & 3,597 & 1,165 & 9,528 & 0,002 & $36,498(3,718 \sim 358,322)$ \\
\hline Doença vascular & 1,280 & 0,574 & 4,979 & 0,026 & $3,597(1,168 \sim 11,071)$ \\
\hline HDL-C & 2,574 & 1,021 & 6,354 & 0,012 & $13,124(1,773 \sim 97,142)$ \\
\hline Escore CHA2DS2-VASc & $-0,441$ & 0,171 & 6,610 & 0,010 & $0,644(0,460 \sim 0,901)$ \\
\hline DAE & $-0,246$ & 0,058 & 18,025 & 0,000 & $0,782(0,698 \sim 0,876)$ \\
\hline DDFVE & $-0,173$ & 0,063 & 7,432 & 0,006 & $0,841(0,743 \sim 0953)$ \\
\hline FEVE & 0,066 & 0,027 & 5,925 & 0,015 & $1,068(1,013 \sim 1,126)$ \\
\hline
\end{tabular}

AVC: acidente vascular cerebral; HDL-C: colesterol de lipoproteina de alta densidade; DAE: diâmetro atrial esquerdo; DDFVE: dimensão diastólica final do ventrículo esquerdo; FEVE: fração de ejeção do ventrículo esquerdo

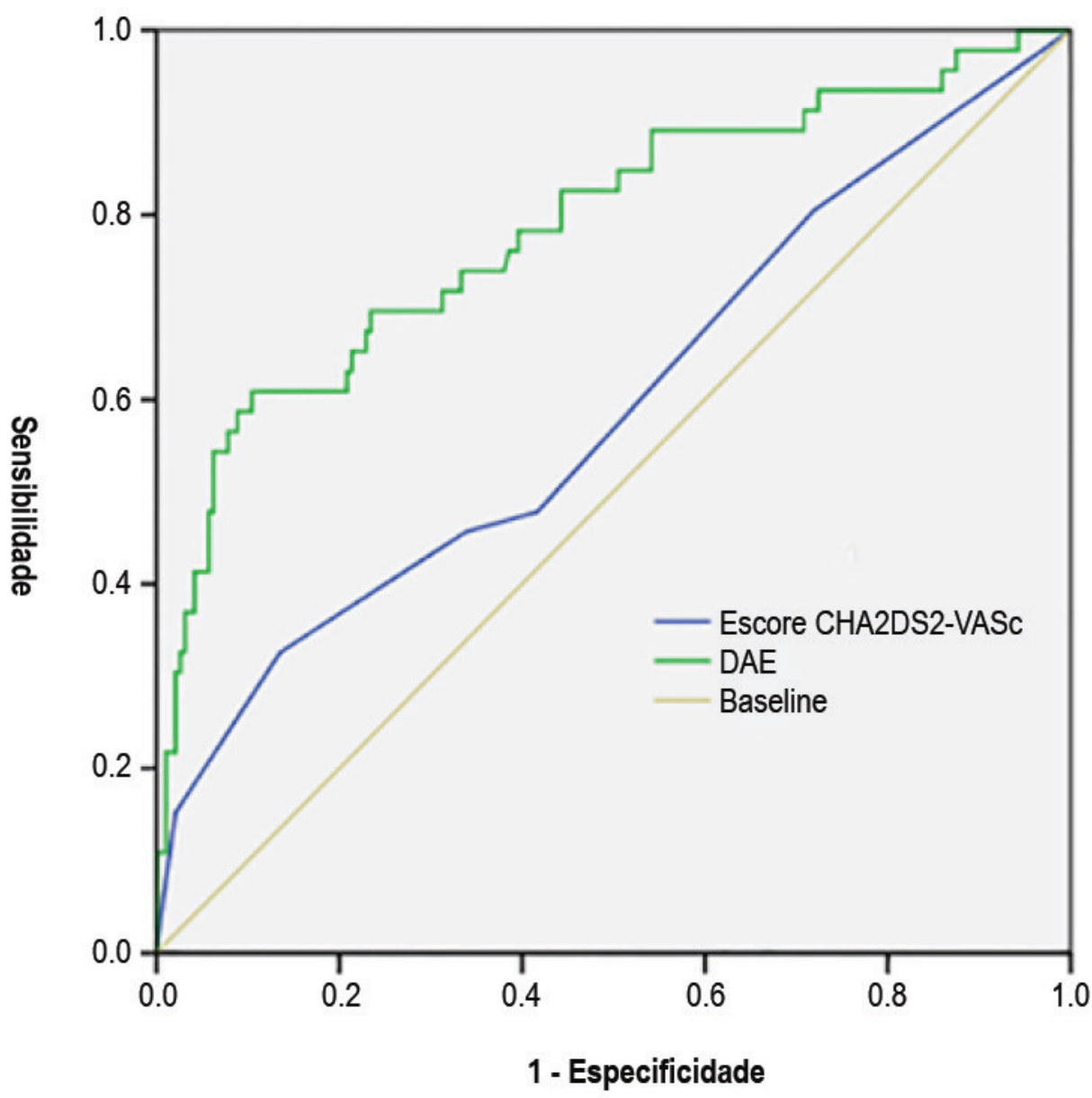

Figura 1 - A análise da área sob a curva ROC revelou que quando o escore CHA2DS2-VASc foi usado para predizer a trombose atrial esquerda/trombose de apêndice atrial esquerdo, a área sob a curva foi de 0,593 (IC 95\%: 0,495-0,690). Quando o escore CHA2DS2-VASc foi $\geq 3$, a sensibilidade e a especificidade foram de $86,5 \%$ e 32,6\%, respectivamente. Quando o DAE foi usado para predizer a trombose atrial esquerda/trombose de apêndice atrial esquerdo, a área sob a curva foi de 0,786 (IC 95\%: 0,704-0,868). Quando o DAE foi >44,17 mm, a sensibilidade e a especificidade foram $89,6 \%$ e 60,9\%, respectivamente. 
Tabela 3 - Efeito do diâmetro do átrio esquerdo sobre o risco de trombose atrial esquerda/trombose de apêndice atrial esquerdo em pacientes com diferentes grupos de CHA2DS2-VASc (mm)

\begin{tabular}{|c|c|c|c|c|c|c|}
\hline \multirow[t]{2}{*}{ Grupo CHA2DS2-VASc } & \multirow[t]{2}{*}{$\mathrm{n}$} & \multicolumn{2}{|c|}{$\begin{array}{l}\text { Trombo de átrio esquerdo/trombo de apêndice } \\
\text { atrial esquerdo }(n, \%)\end{array}$} & \multirow[t]{2}{*}{ OR } & \multirow[t]{2}{*}{$x^{2}$} & \multirow[t]{2}{*}{$\mathrm{p}$} \\
\hline & & $\mathrm{DAE} \geq 44,17$ & $\mathrm{DAE}<44,17$ & & & \\
\hline Escore 0 & 63 & $6 / 12(50,0)$ & $3 / 51(5,9)$ & 8,500 (IC 95\%: 1,856 38.938) & 9,524 & 0,002 \\
\hline Escore 1 & 73 & $11 / 16(68,8)$ & $4 / 57(7,0)$ & 9,797 (IC 95\%: 2,747 34.943) & 15,466 & 0,000 \\
\hline Escore $\geq 2$ & 102 & $11 / 19(57,9)$ & $11 / 83(13,3)$ & 4,368 (IC 95\%: 1,651 11.559) & 9,712 & 0,002 \\
\hline
\end{tabular}

de apêndice atrial esquerdo. A análise da área sob a curva ROC revelou uma área sob a curva de 0,593 (IC 95\%: 0,495-0.690). Quando o escore CHA2DS2-VASc foi $\geq 3$, a sensibilidade e a especificidade foram de $86,5 \%$ e $32,6 \%$, respectivamente. Esses resultados mostraram que, para pacientes com escore CHA2DS2-VASc $\geq 3$, a possibilidade de trombose atrial esquerda/trombose de apêndice atrial esquerdo deve ser altamente alertada. No entanto, este estudo também revelou que quando o escore CHA2DS2VASc era 0 ou 1, a trombose atrial esquerda/trombose de apêndice atrial esquerdo ainda ocorria em 9 e 15 pacientes, respectivamente. Além disso, esses resultados revelaram que, para pacientes de baixo risco com escore CHA2DS2-VASc de 0 ou 1, ainda havia risco de acidente vascular cerebral. Esses resultados sugeriram que o escore CHA2DS2-VASc tem algumas limitações na predição de trombose atrial esquerda/ trombose de apêndice atrial esquerdo.

Um estudo revelou que ${ }^{17}$ alterações morfológicas no átrio esquerdo e no apêndice atrial esquerdo podem aumentar o risco de tromboembolismo em pacientes com FA. Quando a FA ocorre, quanto maior o átrio cardíaco, mais facilmente se forma a trombose. ${ }^{18}$ Neste estudo, comparou-se o DAE em pacientes com FA. Os resultados revelaram um DAE maior no grupo trombose do que no grupo não trombose, sendo um fator de risco independente para trombose atrial esquerda/trombose de apêndice atrial esquerdo. A análise da curva ROC revelou que quando o DAE foi usado para predizer a trombose atrial esquerda/ trombose de apêndice atrial esquerdo, a área sob a curva foi 0,786 (IC de 95\%: 0,704-0,868), e quando o DAE era $\geq 44,17 \mathrm{~mm}$, a sensibilidade e a especificidade eram $89,6 \%$ e $60,9 \%$, respectivamente. Esses resultados revelaram que o tamanho do DAE se correlacionou com a trombose atrial esquerda/trombose de apêndice atrial esquerdo. Portanto, quando o DAE era $\geq 44,17 \mathrm{~mm}$, apresentava boa sensibilidade e especificidade na predição de trombose atrial esquerda/trombose de apêndice atrial esquerdo. Neste estudo, usamos o DAE como o índice para prever trombose atrial esquerda/trombose de apêndice atrial esquerdo. Recentemente, o volume atrial esquerdo tem sido usado como medida do aumento atrial esquerdo. ${ }^{19}$ Esse índice pode ser incluído em estudos futuros. Neste estudo, os pacientes foram estratificados de acordo com o escore CHA2DS2-VASc, a fim de analisar o efeito do DAE na trombose atrial esquerda/trombose de apêndice atrial esquerdo. Esses resultados revelaram que, independentemente de o escore CHA2DS2-VASc ser 0, 1 ou $\geq 2$, um DAE $\geq 44,17 \mathrm{~mm}$ aumentou significativamente o risco de trombose atrial esquerda/trombose de apêndice atrial esquerdo. Esses resultados revelaram que uma avaliação adicional do DAE com base no escore CHA2DS2VASc seria útil para avaliar o risco de trombose atrial esquerda/trombose de apêndice atrial esquerdo e orientar a terapia de anticoagulação.

No entanto, considerando que este é um estudo unicêntrico e com amostra pequena, pode haver algumas deficiências na representatividade da amostra. Portanto, estudos de coorte multicêntricos e com amostras maiores são necessários para esclarecer ainda mais a relação entre o escore CHA2DS2-VASc e o DAE na predição de trombose atrial esquerda/trombose de apêndice atrial esquerdo e orientação da terapia de anticoagulação.

\section{Conclusão}

Em resumo, o escore CHA2DS2-VASc e o DAE estão correlacionados à trombose atrial esquerda/trombose de apêndice atrial esquerdo em pacientes com FA não valvar. Para pacientes com escore CHA2DS2-VASc de 0 ou 1, o tamanho do DAE deve ser considerado. Quando o DAE era $\geq 44,17 \mathrm{~mm}$, o risco de trombose atrial esquerda/trombose de apêndice atrial esquerdo ainda é relativamente alto, sendo necessário conduzir terapias de anticoagulação adicionais.

\section{Contribuição dos autores}

Concepção e desenho da pesquisa, Obtenção de financiamento e Revisão crítica do manuscrito quanto ao conteúdo intelectual importante: Yi-Qiang Y; Obtenção de dados, Análise e interpretação dos dados, Análise estatística e Redação do manuscrito: Zhang Y.

\section{Potencial conflito de interesses}

Os autores declaram não haver conflito de interesses pertinentes.

\section{Fontes de financiamento}

O presente estudo não contou com fontes de financiamento externas.

\section{Vinculação acadêmica}

Não há vinculação deste estudo a programas de pósgraduação. 


\section{Referências}

1. Garwood CL, Korkis B, Grande D, Hanni C, Morin A, Moser LR. Anticoagulation bridge therapy in patients with atrial fibrillation: recent updates providing a rebalance of risk and benefit. Pharmacotherapy. $2017 ; 37(6): 712-24$

2. Wan H, Wu S, Wang J, Yang Y, Zhu J, Shao X, et al. Body mass index and the risk of all-cause mortality among patients with nonvalvular atrial fibrillation: a multicenter prospective observational study in China. Eur J Clin Nutr. 2017;71(4):494-9.

3. Wyrembak J, Campbell KB, Steinberg BA, Bahnson TD, Daubert JP, Velazquez EJ, et al. Incidence and predictors of left atrial appendage thrombus in patients treated with nonvitamin $\mathrm{K}$ oral anticoagulants versus warfarin before catheter ablation for atrial fibrillation. Am J Cardiol. 2017;119(7):1017-1022.

4. Chen YY, Liu Q, Liu L, Shu XR, Su ZZ, Zhang HFet al. Effect of metabolic syndrome on risk stratification for left atrial or left atrial appendage thrombus formation in patients with nonvalvular atrial fibrillation. Chin Med J (Engl). 2016;129(20):2395-2402.

5. Zhao LD, Hong J. New research progress of risk evaluation scores of stroke and bleeding in patients with atrial fibrillation. Chin J Integr Tradit West Med Intens Crit Care. 2017;24(2):213-216.

6. Boyd AC, McKay T, Nasibi S, Richards DA, Thomas L. Left ventricular mass predicts left atrial appendage thrombus in persistent atrial fibrillation. Eur Heart J Cardiovasc Imaging. 2013;14(3):269-75

7. Zhu WG, Xiong QM, Hong K. Meta-analysis of CHADS2 versus CHA2DS2VASc for predicting stroke and thromboembolism in atrial fibrillation patients independent of anticoagulation. Tex Heart Inst J. 2015;42(1):6-15.

8. Almutairi AR, Zhou L, Gellad WF, Lee JK, Slack MK, Martin JR, et al. Effectiveness and safety of non-vitamin Kantagonist oral anticoagulants for atrial ribrillation and venous thromboembolism: A systematic review and meta-analyses. Clin Ther. 2017;39(7):1456-78.

9. Harel Z, Chertow GM, Shah PS, Harel S, Dorian P, Yan AT, et al. Warfarin and the risk of stroke and bleeding in patients with atrial fibrillation receiving dialysis: a systematic review and meta-analysis. Can J Cardiol. 2017;33(6):737-46.
10. Chanda A, Reilly JP. Left atrial appendage occlusion for stroke prevention Prog Cardiovasc Dis. 2017;59(6):626-35

11. Doukky R, Garcia-Sayan E, Patel M, Pant R, Wassouf M, Shah S, et al. Impact of diastolic function parameters on the risk for left atrial appendage thrombus in patients with nonvalvular atrial fibrillation: a prospective stud. J Am Soc Echocardiogr. 2016;29(6):545-553.

12. Lobo R, McCann C, Hussaini A, Meany TB, Kiernan TJ. Left atrial appendage thrombus with resulting stroke post-RF ablation for atrial fibrillation in a patient on dabigatran. Ir Med J. 2014;107(10):329-30.

13. Xie SL, Chen YY, Liu Q, Zhang YJ, Shu XR, Su ZZ, et al. Effect of metabolic syndrome on risk stratification of left atrial thrombus formation in patients with nonvalvular atrial fibrillation. Chin J Cardiac Arrhythm. 2015;19(2):87-93.

14. Fu WW, Li GD, Wang Z. Relationship between high-density lipoprotein cholesterol and left atrial/left atrial appendage thrombus in non-valvular atrial fibrillation patients. J Tianjin Med Univ. 2015;21(2):498-502.

15. Doorn S, Debray TPA, Kaasenbrood F, Hoes AW, Rutten FH, Moons KGM, et al. Predictive performance of the CHA2DS2-VASc rule in atrial fibrillation: a systematic review and meta-analysis. J Thromb Haemost. 2017:15(6):1065-77.

16. Ma JW, Ma XH, Cui LJ, Li Z. The predictive value of CHADS2 and CHA2DS2VASc score in left atrial or left atrial appendage thrombus in patients with non-valvular atrial fibrillation. Tianjin Med J. 2015;43:304-8

17. Qi SY, Tian Y, Shi L, Wang YJ, Lu XY, Chen RM, et al. Left atrial appendage morphology and thromboembolic risk in patients with atrial fibrillation and CHADS2 score $\leq 1$ : a case-control study. Chin J Cardiac Arrhythm. 2015; 19:94-98.

18. Taina M, Vanninen R, Hedman M, Jäkälä P, Kärkkäinen S, Tapiola T, et al Left atrial appendage volume increased in more than half of patients with cryptogenic stroke. PLoS One. 2013;8(11):e79519.

19. Arsanjani R, Flint N, Beigel R, Khachatryan T, Shalev A, Shturman A, et al Comparison of accuracy of left atrial area and volume by two-dimensional trans-thoracic echocardiography versus computed tomography. Am J Cardiol. 2019;123(7):1180-4. 